\title{
The New Type of Transport on Deep Mines and Open Pits - Skip Pneumatic Lifting Equipment for Transportation of the Rock Mass
}

\author{
A. Taranov, Y. Nikolaev and G. Tatkeeva
}

\begin{abstract}
The article is devoted to determining the maximum loads acting on the guide roller devices of skips in the trunk of mine and quarry overpass lifting equipment necessary to develop methods for calculating the guiding devices.
\end{abstract}

Index Terms-mine, open pit, transport, lifting equipment, pneumatic, skip.

\section{INTRODUCTION}

There are two basic ways of mining in the mining industry: open pit and underground. Underground mining less profitable, however, it is more environmentally friendly. In addition, this method is essentially inevitable due to the fact that the major mineral reserves are at greater depths and in the future will need to solve the problems of development of mineral deposits deep mines. The development of the mining sector and the deepening of mining operations require a new type of transport, justified and developed in the works of M.D. Bogatyrev, V.I. Kiselev, N.G. Kartaviy, A.S. Saginov, S.N. Nechyporenko, O.M. Piskunov, Y.A. Nikolaev - skip pneumatic lifting equipment designed to reduce the cost of transportation of the rock mass.

\section{ANALYSIS OF Mine SPLI WORK}

The cycle of operation of pneumatic lifting equipment consists in the following (Figures 1 and 2). Skips 4, 5 are loaded at the lower end of the mine from dispensers 5,7 jet of rock mass falling from a height of more than ten meters in a skip and has a significant dynamic loads on the bottom and sides of the latter. After loading the skip is included working body (turbo blower) 1 and compressed air begins to flow under the bottom of the skip. When a certain overpressure skip is accelerated and begins to accelerate to the steady speed, determines the performance blowers.

The main part of the way to the top mark of the mine skips moves at a constant speed, and when approaching the upper stop device 6, 9 is inhibited due to discharge of the compressed air to the atmosphere through the air valves 9 and 10. After entering the mine skip 4 in abutment device 6 it is unloaded through the side walls into the hopper 7 , and 5 of career skip installation, after fixing in the upper rotary device 9 , bends and unloaded into the hopper 8 .

A. Taranov, Y. Nikolaev and G. Tatkeeva they all are with the Karaganda State Technical University, Bulvar Mira, Karaganda, Kazakhstan
All the time of unloading the skip 4 mine pneumatic lifting installation it is supported by positive pressure in the cavity of the barrel 3. After unloading the skip 5 last of career installation returns to its original position, stops are removed and skip 5 under the action of gravity starts to fall down by squeezing under a cushion of air.

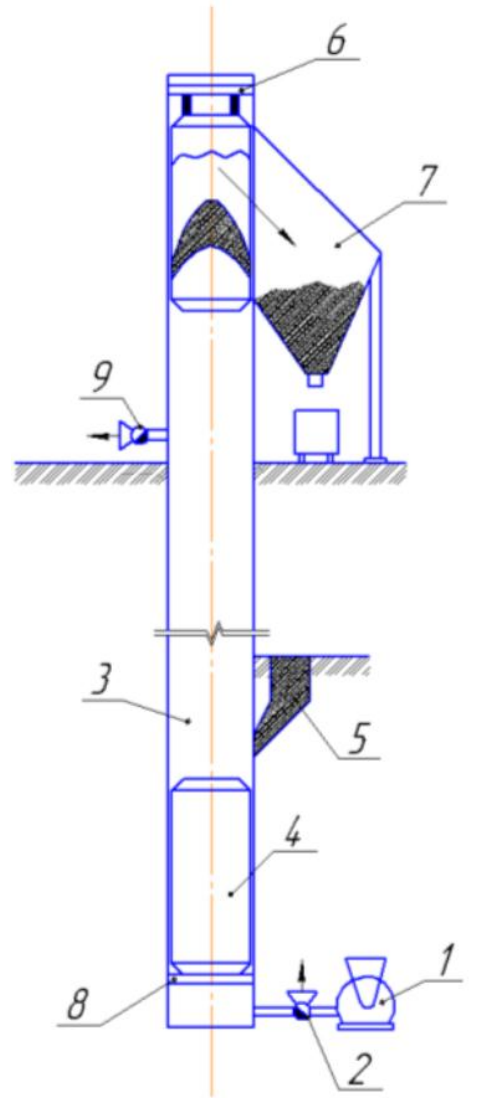

Fig. 1: The structural layout of mine SPLI: 1 - working body (turbo blower); 2, 9 - air lock; 3 - overpass with the guide and sealing devices; 4 -skip; 5 - dispenser; 6 -the upper stop; 7 - unloading hopper; 8 - the lower landing device. 


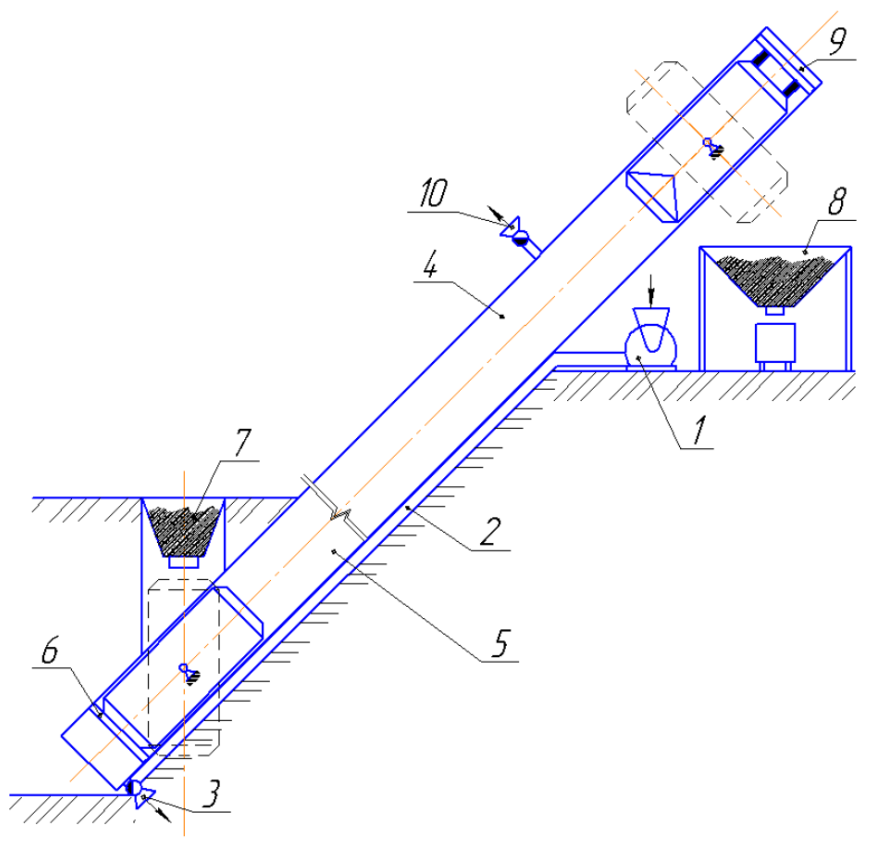

Fig. 2: The structural layout of SPLI open pit: 1 - working body (turbo blower); 2 - air pipe; 3, 10 - air lock; 4 - overpass with the guide and sealing devices; 5 - skip; 6 - lower rotating part of the overpass; 7 dispenser; 8 - unloading hopper; 9 - the upper rotating part of the overpass with a focus.

The lowering speed of skips 4 and 5 and their landing on the lower landing gear 6, 8 is regulated by air locks 2 and 3. The main and most crucial element of mine and career pneumatic lift systems are rails movement the skip device performance depends on the reliability and durability of these plants.

However, the task of creating the guide devices of skips for mines and quarries paid insufficient attention has hindered the implementation of these machines in production. Therefore there is a need to study and selection of the parameters guiding devices of skips of mine and career pneumatic lifting equipment. Large problem of determining the parameters of guiding devices, one of the main elements of these systems, is not sufficiently developed and needs to be resolved, as the reliable operation of skip pneumatic lift systems without solving this problem is impossible. To do this, you must develop a methodology for calculating the guiding devices of mine and career pneumatic lifting equipment, as one of the main elements of these installations. In this regard, the choice of parameters and the design procedure of guiding devices of skips of mine and career pneumatic lifting equipment, is an actual problem. According to the kinematics of the pneumatic lifting equipment, the following basic operating modes guiding devices: - loading the skip; - the skip entry in the guide device with maximum acceleration; - motion of the skip in the guide device with maximum acceleration; - the skip entry in the guide device with a maximum speed; - uniform motion of the skip in guiding devices with a maximum speed; - unloading the skip. Under each mode guidance devices are subjected to static and dynamic loads (Figures 3 and 4), representing different kinds of danger. Reaction of rollers depend on how far from the center axis of the vessel its mass is. Therefore, when designing pneumatic lifting equipment is necessary to ensure such conditions that its center of mass is as close as possible to the axis of symmetry. But in reality, this condition is very difficult to provide because of the uneven loading.
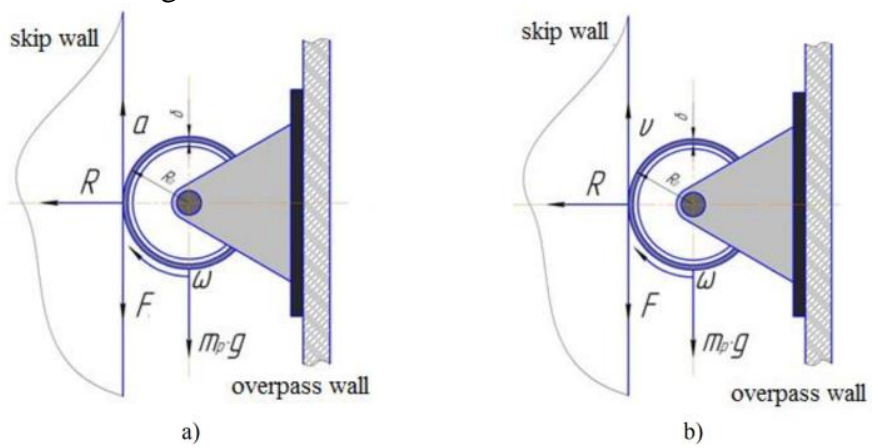

Fig. 3: Scheme of loads guiding devices pneumatic lifting equipment when rolling the skip on rollers: a) the movement of the skip to guide devices with maximum acceleration; b) uniform motion of the skip in guiding devices with a maximum speed.

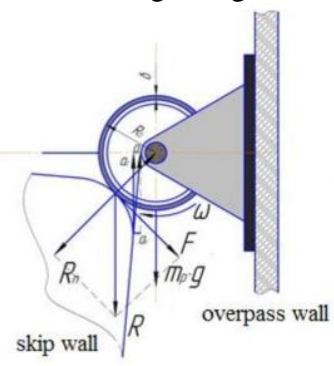

a)

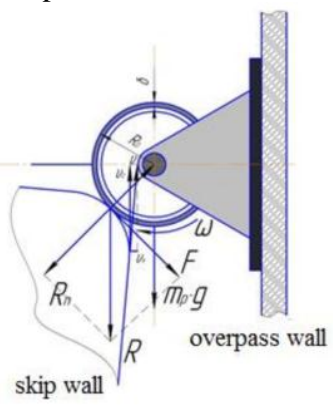

b)
Fig. 4: Scheme of loads guiding devices: a) the skip entry in the guide device with maximum acceleration; b) the skip entry in the guide device with a maximum speed.

In addition, it is necessary to take into account the dynamic processes when adjusting the speed of the vessel and the conditions for its entry in the guide roller devices. The maximum values of reaction of rollers at a coal mine pneumatic lift installation depends only on the diameter (the skip-duty) and practically do not depend on the thickness of the wall of the skip. For ore mine pneumatic lifting installation with thicker walls the skip observed a significant reduction reaction of rollers by increasing the wall thickness of the skip. By increasing the diameter of the intensity of the reduction reaction the skip of rollers weakened. The maximum values of reaction of rollers and coal and ore of career pneumatic lift installation depend not only on the diameter (the skip load capacity) and the wall thickness of the skip. By increasing the diameter of the intensity of the reduction reaction the skip of rollers weakened. The analysis of the results of a numerical calculation using licensed software shows that determines the load in determining the design parameters of the skip, as a rule, local external forces generated by reactions of rollers guiding devices $[1,2]$. To solve the problem of determining the dynamic interaction with the walls of the guide devices the skip (career overpass) developed the design scheme and a mathematical model of the motion of skips of mine and career pneumatic lift systems (Figures 5 and 6). A system of differential equations of motion the skip in the guide device. Unlike the previously known mathematical models [3], the resistance in the guide devices are recorded according to the deformation of the roller over time. The study parameters and guiding devices pneumatic mine lifting 
installation during periods of unsteady motion the skip, as well as the regulation of its speed accomplished using a mathematical model, which is a system of seven differential equations that describe the motion the skip along the axis of the shaft. This mathematical model is based on the calculation scheme shown in Figure 5.
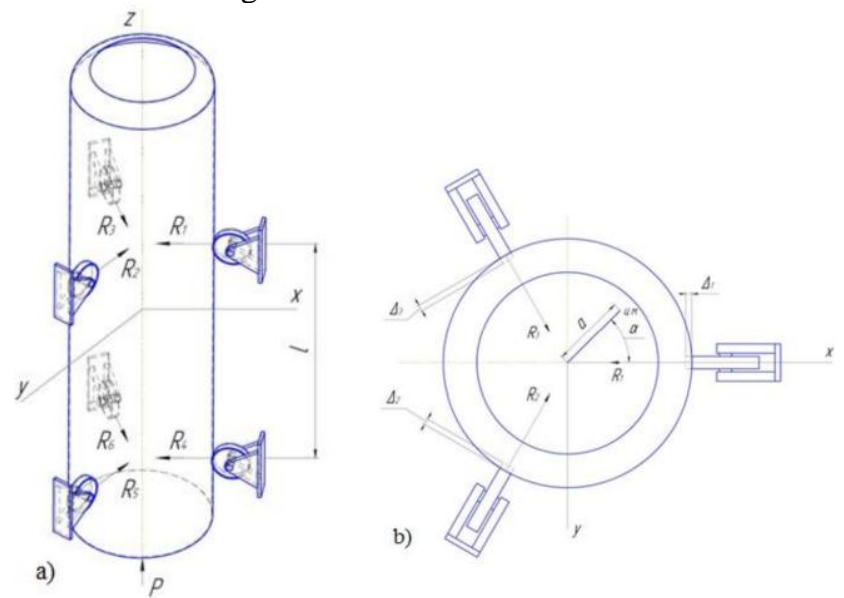

Fig. 5: Design scheme of mine pneumatic lifting installation: a) general view; b) cross-section.

At constant pressure vessels under $P=$ const system of differential equations goes to the following form (1):

$$
\left\{\begin{array}{l}
\frac{d^{2} z}{d t^{2}}=\frac{1}{M}\left[\left(P-P_{a}\right) \cdot S_{c}-F-F_{f r . y y}-\frac{k_{f r} \cdot C}{R_{0}} \cdot\left(\Delta_{1}+\Delta_{2}+\Delta_{3}+\Delta_{4}+\Delta_{5}+\Delta_{6}\right)\right] \\
\frac{d^{2} \Delta_{1}}{d t^{2}}=-\frac{1}{M}\left(C \cdot \Delta_{1}+\beta \frac{d \Delta_{1}}{d t}\right) ; \frac{d^{2} \Delta_{2}}{d t^{2}}=-\frac{1}{M}\left(C \cdot \Delta_{2}+\beta \frac{d \Delta_{2}}{d t}\right) ; \\
\frac{d^{2} \Delta_{3}}{d t^{2}}=-\frac{1}{M}\left(C \cdot \Delta_{3}+\beta \frac{d \Delta_{3}}{d t}\right) ; \frac{d^{2} \Delta_{4}}{d t^{2}}=-\frac{1}{M}\left(C \cdot \Delta_{4}+\beta \frac{d \Delta_{4}}{d t}\right) ; \\
\frac{d^{2} \Delta_{5}}{d t^{2}}=-\frac{1}{M}\left(C \cdot \Delta_{5}+\beta \frac{d \Delta_{5}}{d t}\right) ; \frac{d^{2} \Delta_{6}}{d t^{2}}=-\frac{1}{M}\left(C \cdot \Delta_{6}+\beta \frac{d \Delta_{6}}{d t}\right) ;
\end{array}\right.
$$

where $Z$ is current coordinate of movement the skip measured from the mark of the barrel bottom, m; $M$ is the total mass of the payload and the body of the skip, kg; $P$ is current absolute air pressure in the cavity beneath the vessel barrel, $\mathrm{Pa} ; P_{a}$ is the current value of the atmospheric pressure, $\mathrm{Pa}$;

$S_{c}$ is sectional area of the skip, $m^{2} ; F=M \cdot g$ is useful resistance force, $\mathrm{N} ; F_{f r . y y}$ is resistance force the skip in stem sealing devices, $\mathrm{N} ; R_{i}=\frac{k_{f r} \cdot C}{R_{0}} \cdot \Delta_{i}$ is reaction of rollers of guiding devices, $\mathrm{N} ; k_{f r}$ is coefficient of rolling friction, $\mathrm{N}$; $C$ is rigidity of guiding devices; $R_{0}$ is radius of the roller of guide device, $\mathrm{m} ; \Delta_{1}, \Delta_{2}, \Delta_{3}, \Delta_{4}, \Delta_{5}, \Delta_{6}$ is deformation of rollers of guiding devices; $a$ is the displacement of the center of mass of the laden the skip; $\alpha$ is the angle between the $\mathrm{X}$ axis and the direction of displacement of the center of mass; $\beta$ is viscous friction coefficient.

Under $\alpha=0$ and $P=$ const (2):

$$
\left\{\begin{array}{l}
\frac{d^{2} z}{d t^{2}}=\frac{1}{M} \cdot\left[\left(P-P_{a}\right) \cdot S_{c}-F-F_{f r \cdot y y}-2 \cdot \frac{k_{f r} \cdot C \cdot x}{R_{0}}\right] \\
\frac{d^{2} x}{d t^{2}}=-2 \cdot \frac{1}{M} \cdot\left(C \cdot x+\beta \frac{d x}{d t}\right)
\end{array}\right.
$$

The resultant of the research design scheme of mine pneumatic lifting installation and bears the highest possible operating forces is the basis for design choice guiding devices, calculation of stresses acting in their elements by using the mathematical apparatus of the finite element program ANSYS et al., the selection structural materials and the disc rim roller, testing the strength and stability. The above mathematical model and its numerical solution results allow procedures to develop an algorithm for calculating the parameters guiding devices mine pneumatic lifting equipment. To solve the problem of determining the dynamic interaction with the walls of the guide devices career skip developed analytical model and mathematical model of motion skips career pneumatic lifting equipment. A system of differential equations of motion skip in the guide device. Unlike the previously known mathematical models resistance guiding devices recorded in the roller strain depending on time. The study parameters and guiding devices career pneumatic lifting mechanism during periods of unsteady motion skip, as well as the regulation of its speed accomplished using a mathematical model, which is a system of seven differential equations that describe the motion skip along the career of an overpass. This mathematical model is based on the calculation scheme shown in Figure 6. At constant pressure vessels under $P=$ const $(3)$ :

$$
\left\{\begin{array}{l}
\frac{d^{2} z}{d t^{2}}=\frac{1}{M}\left[\left(P-P_{a}\right) \cdot S_{c}-F \cdot \sin \beta_{c}-F_{f r . y y}-\frac{k_{f r} \cdot C}{R_{0}} \cdot \sum_{i=1}^{n} \Delta_{i}\right] \\
\frac{d^{2} \Delta_{1}}{d t^{2}}=-\frac{1}{M}\left(C \cdot \Delta_{1}+\beta \frac{d \Delta_{1}}{d t}\right) ; \frac{d^{2} \Delta_{2}}{d t^{2}}=-\frac{1}{M}\left(C \cdot \Delta_{2}+\beta \frac{d \Delta_{2}}{d t}\right) ; \ldots \\
\ldots ; \frac{d^{2} \Delta_{n}}{d t^{2}}=-\frac{1}{M}\left(C \cdot \Delta_{n}+\beta \frac{d \Delta_{n}}{d t}\right)
\end{array}\right.
$$

where $\beta_{c}$ is the angle of the career of an overpass to the horizon; $\mathrm{n}$ is the number of guiding devices are simultaneously in contact with loader.

The above mathematical model and the results of its numerical solution allowed us to develop an algorithm for calculating the parameters of procedures guiding devices career pneumatic lifting equipment.

Numerical study of the dynamics of motion skip pneumatic lifting equipment was carried out by a licensing system MatLab (Matrix Laboratory), which is an interactive system for performing engineering and scientific calculations, and is focused on work with arrays of data $[4,5]$. The system makes it easy to exchange information with the word processor Microsoft Word, in particular to carry any text and images to the clipboard or read text strings from the clipboard as executable instructions. The system MatLab are special means of solving the Cauchy problem for systems of ordinary differential equations given in explicit $\frac{d x}{d t}=F(t, x)$, and in the implicit $M \frac{d x}{d t}=F(t, x)$. Here M-matrix - the so-called ODE solver (solver ODE), allows the user to select the method, the initial conditions, etc. 


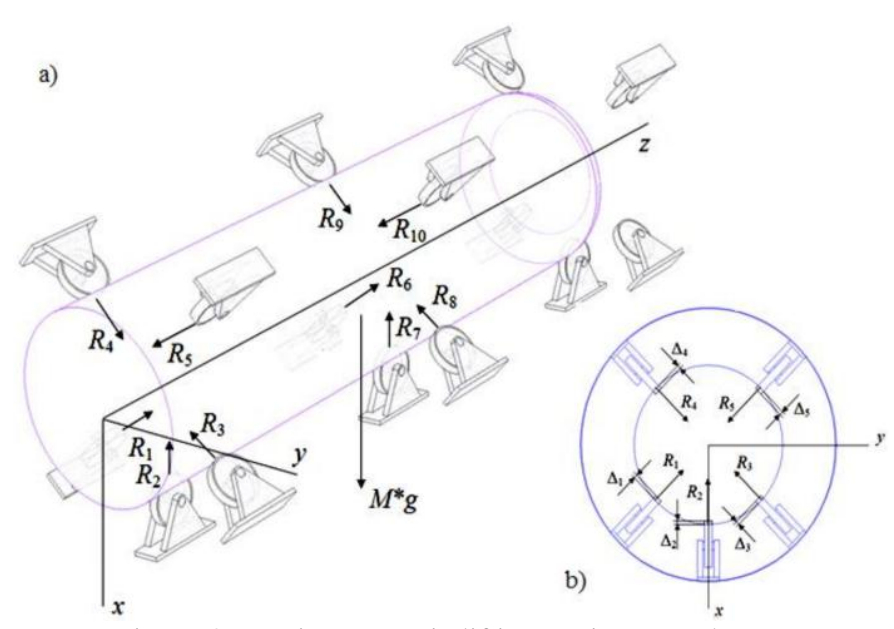

Fig. 6: Open pit pneumatic lifting equipment scheme:

a) general view; b) cross-section.

To solve ODE systems in MatLAB various methods implemented. Their implementation called ODE solvers. Solvers implement the following methods for solving systems of differential equations:

- Ode45 is one-step explicit Runge-Kutta 4th and 5th order. This is a classic method recommended for the initial sample solutions. In many cases, it gives good results;

- Ode23 is one-step explicit Runge-Kutta 2nd and 4th order. In moderate rigidity of the system of ODE and low accuracy requirements, this method can provide a gain in speed solutions;

- Ode 133 is multi-step method of Adams-Moulton-Bashvorta variable order. The adaptive method that can provide high accuracy solutions;

- Ode $15 s$ is multi-step method of variable order (from the first to the fifth, the default is 5), using the formula of numerical differentiation. This adaptive method, it should be applied, if the solver Ode 45 not provide solutions;

- Ode23s is one-step method, which uses a modified Rosenbrock formula of order 2. Can provide a high computational speed with low accuracy;

- Ode23t is trapezium method with interpolation. This method gives good results in solving problems describing oscillators with nearly harmonic output signal;

- Ode23tb is implicit Runge-Kutta method at the beginning of the solution and the method using the inverse of differentiation formula of order 2 in the future. At low precision, this method may be more efficient than Ode15s.

All solvers can solve systems of equations of the explicit form $y^{\prime}=F(t, y)$. Solvers Ode15s, Ode23s, Ode23t, Ode23tb can solve the equations of implicit form $M y^{\prime}=F(t, y)$. All of these methods are incorporated in a means of solving differential equations Simulink. Solution of the mathematical model in the application program Matlab Simulink is shown in Figure 7.

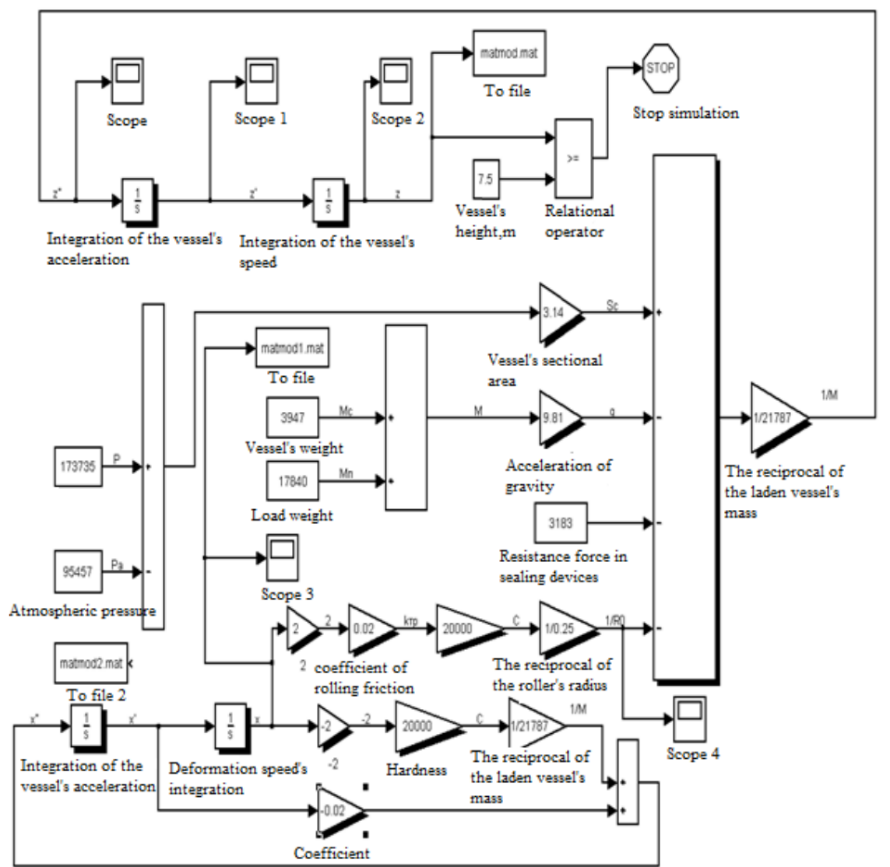

Fig. 7: Solving mathematical model of pneumatic lifting equipment in the application program Matlab Simulink.

As described in the following functions for the solution of systems of differential equations, the following notation and rules: $F$ is the name of the ODE-file, that is a function of $t$ and $y$, which returns a column vector; tspan is the vector determining the integration interval $[t 0$ tfinal $]$. To obtain solutions at specific times $t 0, t 1 \ldots, t$ final (arranged in order of decreasing or increasing), you must use tspan $=[t 0 t 1 \ldots t$ final $] ; y 0$ is the vector of initial conditions; options is argument to create functions odeset; $p 1, p 2 \ldots$ is Arbitrary parameters passed to the function $\mathrm{F} ; \mathrm{T}, \mathrm{Y}$ is solution matrix $\mathrm{Y}$, where each line corresponds to the time returned in the column vector $\mathrm{T}$. We now turn to the description of functions for solving systems of differential equations (we denote the concept solver one of the possible numerical methods for solving ODE: ode45, ode23, ode113, ode $15 s$, ode $23 s$, ode $23 t$, ode $23 t b):[T, Y]=$ solver (' $F$ ' , tspan, $y 0)$ integrates the system of differential equations of the form $y^{\prime}=F(t, y)$ the interval tspan with the initial conditions $y 0$. ' $\mathrm{F}$ ' - a string containing the name of the ODEfile. The function $\mathrm{F}(\mathrm{t}, \mathrm{y})$ must return a column vector. Each row in the array of solutions $\mathrm{Y}$ corresponds to the time returned in the column vector T. $[T, Y]=$ solver $\left(' F^{\prime}\right.$, tspan, y0, options $)$ gives a solution similar to that described above, but with the parameters determined by the values of the argument options, created with odeset. Commonly used options include permissible value of the relative error RelTol (default $1 e-3$ ) and the vector of allowable values of the absolute error AbsTol (all the components default to $1 e-6 ;[T, Y]=\operatorname{solver}\left({ }^{\prime} F^{\prime}\right.$, tspan, $y 0$, options , $p 1, p 2, \ldots$ ) provides a solution similar to that described above, by passing additional parameters $p 1, p 2$ ... Use options $=[]$, if no options are specified. ODE solver enables the production of solutions of systems of $n$ equations. ODE system can be either homogeneous or heterogeneous. From the graphs, we can draw conclusions about the nature of dynamical fluctuations skip to the transverse axis of the shaft direction (Figure 8,9). Periodic fluctuations are damped, where 
damping is exponentially damping factor for mechanical parameters of the dynamic system "skip - guiding device" has different meanings, which can be calculated from the equation. The experimental model velocities and accelerations in the transverse direction are used to determine the loads on the rollers and the guide devices powers are compared with the values obtained from the mathematical model. Revealed that the maximum dynamic loads on the guide device by pneumatic skip lifting equipment as it moves are determined depending on the weight of the loaded skip, the shift of its center of mass, the angle of the overpass to the horizon and the coefficient of dynamic $(3,4)$ :

$$
\begin{gathered}
R_{i}=\left(1+k_{o} \cdot V\right) \cdot M \cdot g \cdot \frac{a}{l} \\
R_{i}=\frac{\left(1+k_{o} \cdot V\right) \cdot M \cdot g \cdot \cos \beta_{c}}{n},
\end{gathered}
$$

where $V$ - the velocity of the skip, $\mathrm{m} / \mathrm{s} ; k_{o}$ is stiffness coefficient overpass; $a$ is the displacement of the center of mass of the laden skip; $l$ is the distance between adjacent tiers of guiding devices; $k_{d}=1+k_{c} \cdot V$ is dynamic factor.
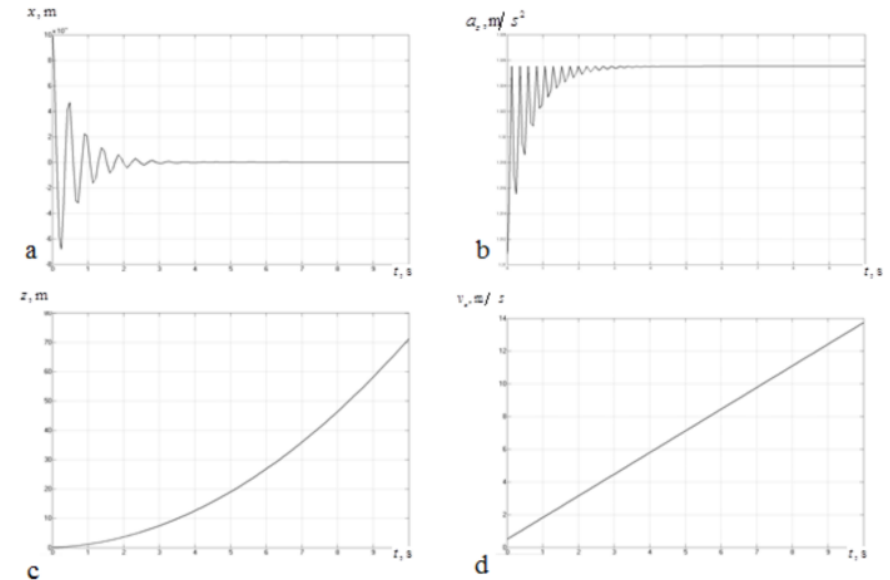

Fig. 8: Results of the solution of the mathematical model of pneumatic lifting equipment in the application program Matlab Simulink:

a) dependence of the deformation roller from time to time;

b) dependence of the acceleration skip from time to time;

c) dependence on the time of travel Skip;

d) dependence of the rate on the time skip.

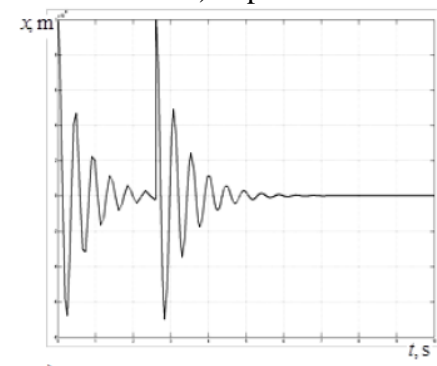

a)

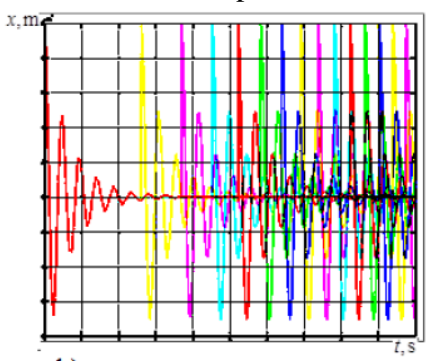

b)
Fig. 9: Walkthrough skip several tiers guiding devices: a) passage of two tiers; b) passage of fifteen tiers.

\section{CONCLUSION}

Developed and researched mathematical model of the motion skip in systems of quarry pneumatic lifting equipment and mine have established patterns of interaction "skip - guiding devices". It was found that the system of "skip - guiding devices" most loaded at the time of entry unevenly loaded skip stage vanes. Revealed that the maximum dynamic loads on the guide device by pneumatic skip lifting equipment as it moves are determined depending on the weight of the loaded skip, the shift of its center of mass, the angle of the overpass to the horizon and the coefficient of dynamic. The adequacy of the emerging dynamic loads on the roller guide device is confirmed by experimental research models skip pneumatic lifting equipment.

\section{REFERENCES}

[1] Zhautikov B. A. Analysis of efforts on housing skip open pit pneumatic lifting equipment Bulletin of the National Engineering Academy of the Republic of Kazakhstan 20063 P. 38-42.

[2] Nikolaev Yu A and Rudaya R P 1981 A mathematical model of pneumatic shaft skip lifting equipment Proceedings of the universities Mining Journal 10 P. 88.

[3] Chen K, Dzhiblin P and Irving A MATLAB's mathematical research Moscow: Mir, 2001 P. 346

[4] Chernih I.V. SIMULINK: creation environment engineering applications 2003 Moscow: DIALOG-MIFI, 2003 P. 496

[5] Porshnev S.V. Using Simulink package to describe the dynamics of oscillating systems with several degrees of freedom Electronic scientific journal «Bulletin of Omsk State Pedagogical University» 2006 P.1-5. 